(C) 2016 IEEE. Personal use of this material is permitted. Permission from IEEE must be obtained for all other uses, in any current or future media, including reprinting/republishing this material for advertising or promotional purposes, creating new collective works, for resale or redistribution to servers or lists, or reuse of any copyrighted component of this work in other works. 


\title{
Cardiovascular Fitness Strengthening Using Portable Device
}

\author{
Hamzah Alqudah*, Kai Cao,Tao Zhang, Azzam Haddad, Steven Su, Branko Celler and Hung T. Nguyen
}

\begin{abstract}
The paper describes a reliable and valid Portable Exercise Monitoring sysetm developed using TI eZ430-Chronos watch, which can control the exercise intensity through audio stimulation in order to increase the Cardiovascular fitness strengthening.
\end{abstract}

\section{INTRODUCTION}

Exercising plays a vital role in improving and protecting the human being health; many diseases can be avoided by just exercising regularly, those diseases such as cancer, blood pressure, heart problems and diabetes. Heart Rate (HR) and Oxygen Uptake $\left(\mathrm{VO}_{2}\right)$ are key indicators of functional health status; their measurements can aid early detection of cardiac diseases [1], [2]. The cardiovascular fitness is defined as the ability of the heart and lungs to supply oxygen-rich blood to the working muscle tissues and the ability to use oxygen to produce energy for movement [3]. Cardiovascular fitness is measured as the amount of oxygen transported in the blood and pumped by the heart to the working muscles and as the efficiency of the muscles to use the oxygen. Regular exercise can increase the cardiovascular fitness as the heart becomes more efficient at pumping oxygen-rich blood to working muscles and body tissues [4]. Increasing the cardiovascular fitness means increasing the capability of the heart and the rest of the cardiovascular system in supplying oxygen and energy to the body.

The training protocol consists of three major phases; that is a warm up, exercise and cools down. Warm up prepares the body for more intense exercise by improving blood flow to the heart, increasing the muscle temperature and protecting against injury through improved flexibility of muscles [5]. The second phase in training protocol is the exercising; the main characteristics of this phase include the intensity, duration, frequency and mode of exercise [6]. Cooling down is the last phase of the training protocol and is defined as the phase that bring the body back to its normal physiological level after fast, vigorous exercise or activity by gradually slowing the pace of activity or by doing gentle exercises or stretches [7]. Any training or workout such as running, swimming or cycling that involves high intensity training session with resting periods is called

*Hamzah Alqudah, Kai Cao, Tao Zhang, Azzam Haddad, Steven W. $\mathrm{Su}$, and Hung. T. Nguyen are with the Centre for Health Technologies, Faculty of Engineering and Information Technology, University of Technology, Sydney, New South Wales, Australia. Branko Celler is with Faculty of Engineering, University of New South Wales, Australia. (email: (Hamzah.M.Alqudah, Kai.Cao-1, Tao.Zhang-4) @student.uts.edu.au, Azzam.Haddad@ resmed.com.au (Steven.Su, Hung.Nguyen) @uts.edu .au, B.Celler@unsw.edu.au interval training protocol. The interval training protocol has proven to build up and strengthen the athletes cardiovascular system [8]. It can usually be noticed that long distance runners are performing the interval training protocol as well as footballers. This newly developed wearable exercise monitoring system has been customized to suit Interval Training.

The Portable Exercise Monitoring System was implemented by using the eZ430-Chronos watch [9] from Texas Instruments, BM-CS5 wireless chest strap [10] from BM Innovations and $\mathrm{K}^{2} \mathrm{~b}^{2}$ [11] from COSMED. The eZ430-Chronos watch is a flexible and powerful development tool, which can integrate heart rate monitor as one of physiological sensors by using low power consumption wireless communications. The BM-CS5 is using BlueRobin ${ }^{\mathrm{TM}}$ data transmission technology to measure and transmit the heart rate values wirelessly.

$\mathrm{K} 4 \mathrm{~b}^{2}$ is the first portable system for pulmonary gas exchange measurement with true breath-by-breath analysis, $\mathrm{K} 4 \mathrm{~b}^{2}$ has previously been reported to be valid, accurate and reliable [12], [13]. $\mathrm{K} 4 \mathrm{~b}^{2}$ has been used in this study to compare the measured HR values with that one from eZ430Chronos watch and it has been found that they are nearly identical under proper pre-signal processing, which means using the proposed portable exercise monitoring system is valid, reliable and cost effective in building cardiovascular fitness. Figure 1 show various devices used in this study.

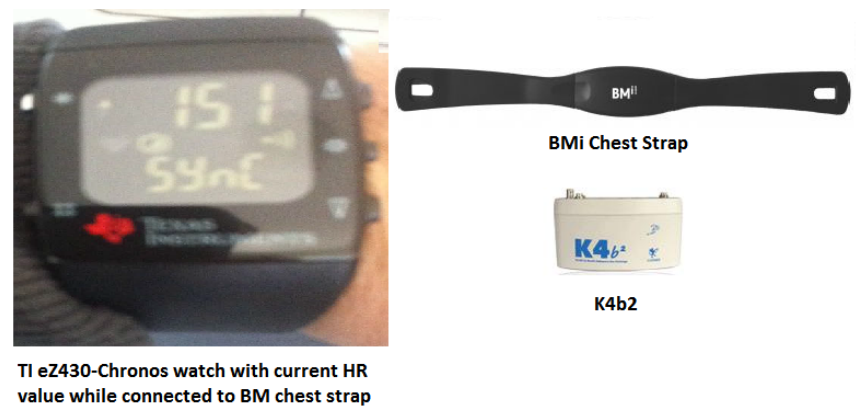

Fig. 1. Wearable Exercise Training System

The UTS Human Research Ethics Committee (UTS HREC 2009000227) approved the study.

\section{THE WEARABLE EXERCISE TRAINING SYSTEM}

The Portable and Wearable Exercise Monitoring System consists of two parts, the eZ430-Chronos watch and the 
BM-CS5 chest strap. However, $\mathrm{K} 4 \mathrm{~b}^{2}$ has been used in this study to verify and compare the HR values. The interval training protocol has been adopted in this study to strengthening the cardiovascular fitness, in this protocol, the exerciser is required to shift between high intensity and low intensity exercise.

The Inbar [14] formula has been used to find out the $\mathrm{HR}_{\max }$ for each participant as following:

$$
\begin{aligned}
H R_{\max } & =205.8-0.685 \times \text { age } \\
& =205.8-0.685 \times 26 \\
& =187.99 \\
& \approx 188
\end{aligned}
$$

So, as we can see that for participant whose age is 26 years old, his $\mathrm{HR}_{\max }$ is approx. $188 \mathrm{bpm}$. The subject must reach $80 \%$ of his $\mathrm{HR}_{\max }$ to guarantee an improvement in the cardiovascular fitness [15].

The proposed system monitors and guides the exerciser through audio stimulation to alter between High Intensity (the subject reaches $80 \%$ of $\mathrm{HR}_{\max }$ ) and Low Intensity (subject reaches $60 \%$ of $\mathrm{HR}_{\max }$ ) exercises in order to build and strengthening his/her cardiovascular system fitness.

\section{A. Subjects}

Five volunteers with different ages and physical characteristics were participated in the experiments; all volunteers were free from any health issues. The subjects physical characters are shown in the table I:

TABLE I

PHYSICAL CHARACTERSTICS OF THE PARTICIPANTS.

\begin{tabular}{|c|c|c|c|}
\hline Subjects & Age (Yrs) & Height $(\mathrm{cm})$ & Weight (Kg) \\
\hline 1 & 25 & 168 & 73 \\
\hline 2 & 27 & 174 & 75 \\
\hline 3 & 30 & 177 & 72 \\
\hline 4 & 33 & 168 & 74 \\
\hline 5 & 26 & 172 & 65 \\
\hline
\end{tabular}

As mentioned earlier, the subject must reach $80 \%$ of his $\mathrm{HR}_{\max }$ to guarantee an improvement in the cardiovascular fitness, and based on Formula (1), the $\mathrm{HR}_{\max }$ have been calculated for all participants along with their High Intensity and Low Intensity HR values as shown in table II:

TABLE II

PARTICIPANTS HR MAX VALUES

\begin{tabular}{|c|c|c|c|c|}
\hline Subjects & Age (Yrs) & $\mathrm{HR}_{\max }$ & $60 \%$ of $\mathrm{HR}_{\max }$ & $80 \%$ of $\mathrm{HR}_{\max }$ \\
\hline 1 & 25 & 189 & 113 & 151 \\
\hline 2 & 27 & 187 & 112 & 150 \\
\hline 3 & 30 & 185 & 111 & 148 \\
\hline 4 & 33 & 183 & 110 & 147 \\
\hline 5 & 26 & 188 & 113 & 150 \\
\hline
\end{tabular}

\section{B. Flowchart}

The flowchart of the proposed health monitoring system running on eZ430-Chronos watch is represented in Figure 2

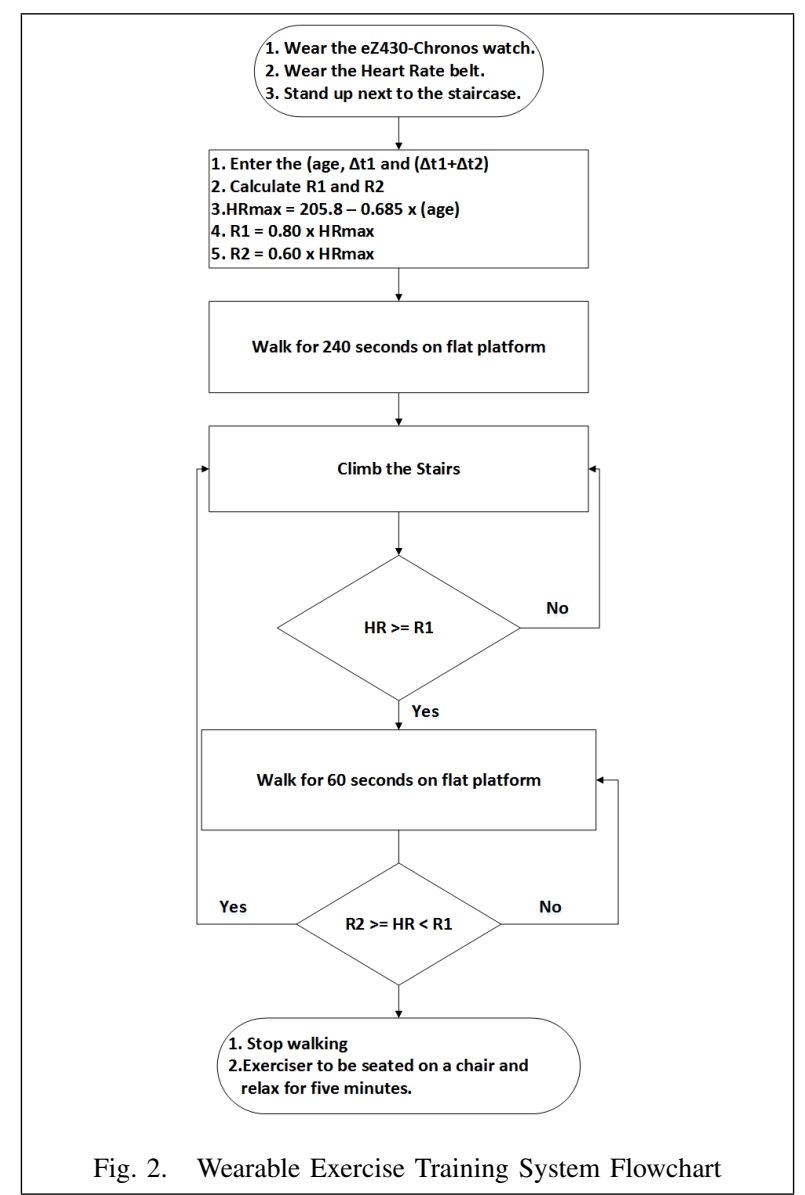

In this instance, after a successful connection between the wearable eZ430-Chronos watch and the BM-CS5 chest strap, the system prompts the exerciser to enter his age and the recommended training zones are computed based on the HR values.

\section{Experiements}

The proposed system guides the exerciser during any exercise whether indoor or outdoor through audio stimulation to switch between high and low intensity exercise to guarantee cardiovascular fitness increase and development.

The proposed system tested on stairs climbing exercise. The experiment starts by asking the subject to wear the ez430-chronos watch on their hand, the BM-CS5 chest strap on their chest, and the $\mathrm{K}_{4} \mathrm{~b}^{2}$ system and mask. The devices will continually measure the HR and the subject is required to act accordingly.

In this experiment, the exercise starts by warming up phase which is walking on a flat platform for 240 seconds; the 
exercising phase starts by climbing the stairs for a specific time, i.e., 60 seconds, or until the subject reaches $80 \%$ of his $\mathrm{HR}_{\max }$. The system will produce an audio tone to notify the subject to alter the exercise intensity from High Intensity to Low Intensity exercise, which is in this case is walking aside on the step for either 60 seconds or until he reaches $60 \%$ of his $\mathrm{HR}_{\max }$, Once this condition becomes true, the system will produce a different audio tone to notify the subject to switch back to High Intensity exercise. High Intensity exercise is climbing the stairs, while walking on the same stair is considered Low Intensity exercise as shown in Figure 3.

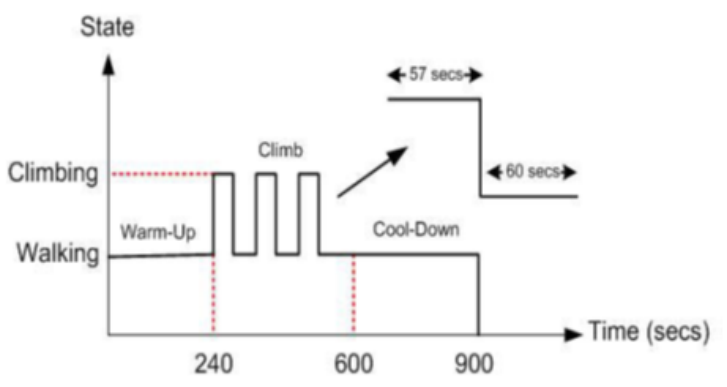

Fig. 3. Walk-Climb-Walk Interval Training Protocol

The subjects has been seated and rested for five minutes after completing the final walk.

Figure 4 shows the $\mathrm{HR}$ and $\mathrm{VO}_{2}$ experimental results for subject 5 under the proposed interval training protocol.
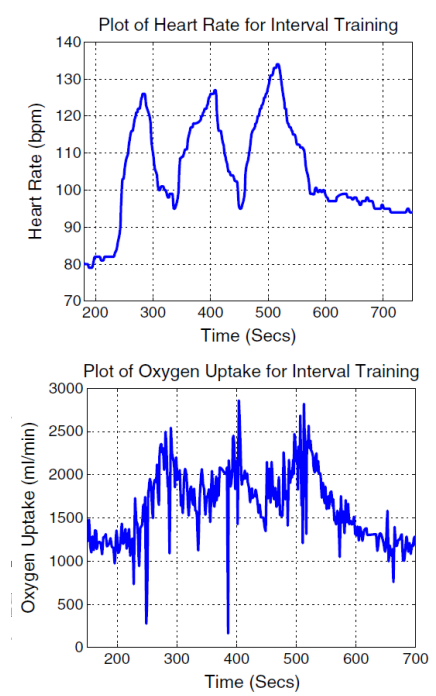

Fig. 4. $\mathrm{HR}$ andVO 2 experimental results for subject 5 under the proposed ITP

\section{Results}

The interval training protocol aim in the study is to develop the cardiovascular fitness system, having this in mind; the exerciser is required to train in a range of $70 \%$ to $80 \%$ of his $\mathrm{HR}_{\max }$, as mentioned earlier, climbing the stairs represent onset stage in the training protocol and walking on the same step is considered as offset period.

A controller with self-adaption feature that can tune the duty cycle $\Delta \mathrm{t}_{1}$ and the period $\left(\Delta \mathrm{t}_{1}+\Delta \mathrm{t}_{2}\right)$ gives the exerciser the opportunity to reach the desired setpoints after a certain number of training sessions as shown in Figure 5

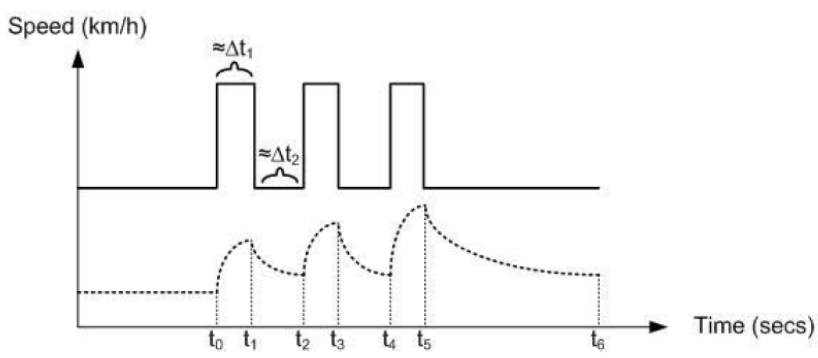

Fig. 5. HR Response During ITP

Figure 6 shows the controller structure that has been implemented in the watch, where the exerciser needs to enter age, $\Delta \mathrm{t}_{1}$ and $\left(\Delta \mathrm{t}_{1}+\Delta \mathrm{t}_{2}\right)$, and based on these values the $\mathrm{R}_{1}$ and $R_{2}$ are calculated, where $R_{1}$ is the onset value and $R_{2}$ is the offset value.

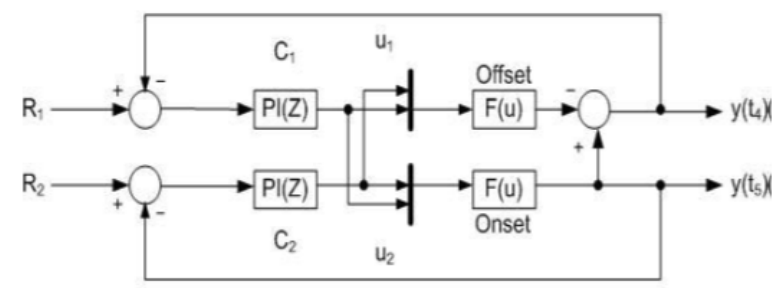

Fig. 6. Controller structure

The watch and its controller will guide the exerciser of how many iteration does he need to reach his setpoints, and the controller will tune up the outputs values until it reaches the desired setpoints. The watch and controller parameters are as shown in table III:

TABLE III

WATCH AND CONTROLLER PARRAMETERS

\begin{tabular}{|c|c|c|c|c|c|c|}
\hline Iteration & Onset Time & Offset time & Period & Duty Cycle & $\mathrm{y}\left({ }_{\mathrm{t}}\right)$ & $\mathrm{y}\left({ }_{\mathrm{t}}\right)$ \\
\hline Ref. & 60 & 60 & 120 & 50 & 113 & 150 \\
\hline
\end{tabular}

In the first iteration, 60 seconds is set to be the onset and offset times. At the end of the first training session, the reading of $\mathrm{HR}$ at $\mathrm{t}_{4}$ and $\mathrm{t}_{5}$ were observed and feed backed to the multi-loop control system to update the controller outputs inputs (the duty cycle and the period). Accordingly, the controller adjusts the time of onset and offset periods 
for the next training session. Figure 7 shows the heart rate response after the first, second and the third iterations.

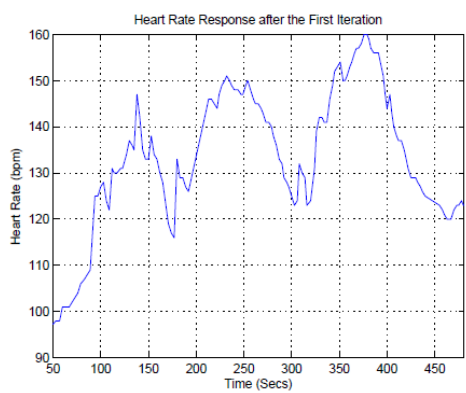

(a) First iteration

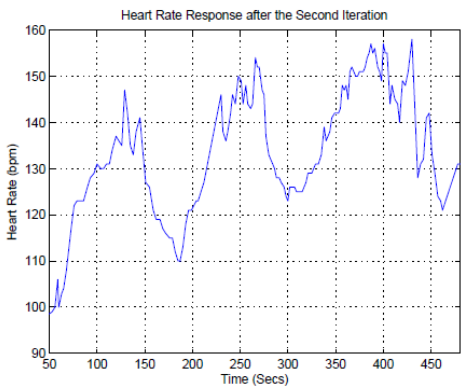

(b) Second iteration

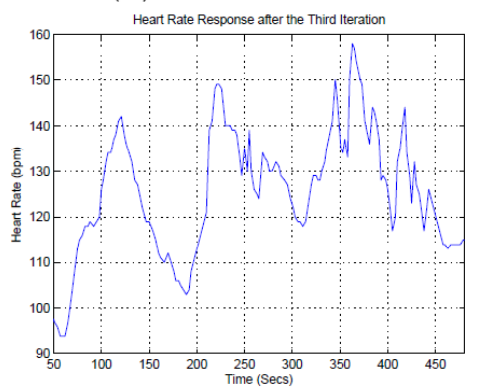

(c) Third iteration

Fig. 7. Subject 5s HR responses under stairs climbing exercise

Table IV shows the watch and the controller parameters after the third iteration:

TABLE IV

WATCH AND CONTROLLER PARRAMETERS AFTER THE THIRD ITERATION

\begin{tabular}{|c|c|c|c|c|c|c|}
\hline Iteration & Onset Time & Offset time & Period & Duty Cycle & $\mathrm{y}\left({ }_{\mathrm{t} 4}\right)$ & $\mathrm{y}\left({ }_{\mathrm{t} 5}\right)$ \\
\hline Ref. & 60 & 60 & 120 & 50 & 113 & 150 \\
\hline 1 & 54.48 & 56.18 & 110.66 & 49.23 & 123 & 160 \\
\hline 2 & 55.52 & 56.59 & 112.11 & 49.52 & 122 & 156 \\
\hline 3 & 56.25 & 56.78 & 113.03 & 49.77 & 118 & 157 \\
\hline
\end{tabular}

We can conclude from the previous table that the exerciser has almost reached to the desired setpoints after only 3 iterations. We kept in mind that after certain number of training sessions, the training capacity of the exerciser will improve, and this might affect the result of his/her HR response.

\section{CONCLUSION}

A prototype of portable health monitoring system has been developed, implemented and tested.

The proposed system allows continuous monitoring of heart rate during any exercise and guides exerciser using audio stimulation to increase or decrease exercise intensity in order to build and strengthening the cardiovascular system based on interval training protocol.

The developed Portable and Wearable Monitoring device and its associated algorithms are technically feasible in various exercise monitoring and regulation related projects. Based on this portable system, recently, a new effective interval training protocol has been proposed and implemented in the Centre of Health Technologies, University of Technology Sydney (UTS). Furthermore, the developed system can be utilized in the control of other automated exercise machines, such as treadmill or bicycle for rehabilitation program and exercise training.

\section{REFERENCES}

[1] R. Acharya, A. Kumar, P. Bhat, C. Lim, N. Kannathal, S. Krishnan et al., "Classification of cardiac abnormalities using heart rate signals," Medical and Biological Engineering and Computing, vol. 42, no. 3, pp. 288-293, 2004.

[2] J. Achten and A. E. Jeukendrup, "Heart rate monitoring," Sports medicine, vol. 33, no. 7, pp. 517-538, 2003.

[3] "Wikipedia, Cardiovascular Fitness," https://en.wikipedia.org/ cardiovascular_fitness.

[4] R. J. Donatelle, Health, the basics. San Francisco: Pearson Education Inc., vol. 6.

[5] I. Champaign, "Essentials of strength training and conditioning," National Strength and Conditioning Association, 2000.

[6] B. L. Williams and Wikins, "Acsm guidelines for exercise testing and prescription," American College of Sports and Medicine, vol. 6, 2006.

[7] "Wikipedia, Cooling Down," https://en.wikipedia.org/cooling_down.

[8] M. J. Gibala, "High-intensity interval training: a time-efficient strategy for health promotion?" Current Sports Medicine Reports, vol. 6, no. 4, pp. 211-213, 2007.

[9] "Texas Instruments, eZ430 Chronos watch," http://http://www.ti.com/ tool/ez430-chronos.

[10] "BM Innovations, BM-CS5 Chest Strap," http://http://www. bm-innovations.com/index.php/chest_straps.html.

[11] “COSMED, K4b2," http://http://www.cosmed.com/en/products/ cardio-pulmonary-exercise-testing/k4-b2-mobile-cpet.

[12] R. Duffield, B. Dawson, H. Pinnington, and P. Wong, "Accuracy and reliability of a cosmed k4b 2 portable gas analysis system," Journal of Science and Medicine in Sport, vol. 7, no. 1, pp. 11-22, 2004.

[13] H. C. Pinnington, P. Wong, J. Tay, D. Green, and B. Dawson, "The level of accuracy and agreement in measures of $\mathrm{f}$ e o 2 , $\mathrm{f}$ e co 2 and $\mathrm{v}$ e between the cosmed $\mathrm{k} 4 \mathrm{~b} 2$ portable, respiratory gas analysis system and a metabolic cart," Journal of Science and Medicine in Sport, vol. 4, no. 3, pp. 324-335, 2001.

[14] O. Inbar, A. Oren, M. Scheinowitz, A. Rotstein, R. Dlin, an R. Casaburi, "Normal cardiopulmonary responses during incremental exercise in 20-to 70-yr-old men." Medicine and science in sports and exercise, vol. 26, no. 5, pp. 538-546, 1994.

[15] C. Balderrama, G. Ibarra, J. De La Riva, and S. Lopez, "Evaluation of three methodologies to estimate the vo $2 \mathrm{max}$ in people of different ages," Applied ergonomics, vol. 42, no. 1, pp. 162-168, 2010. 